\title{
A MASSIVE BLACK HOLE IN THE ACTIVE GALAXY NGC6251
}

\author{
LAURA FERRARESE \\ California Institute of Technology, Pasadena CA, USA \\ HOLLAND C. FORD \\ Johns Hopkins University, Baltimore MD, USA \\ AND \\ WALTER JAFFE \\ Leiden Observatory, Leiden, The Netherlands
}

NGC 6251 is an E2 galaxy with $\mathrm{m}_{B}=13.6 \mathrm{mag}$ (RC3) and heliocentric velocity $7400 \mathrm{~km} \mathrm{~s}^{-1}\left(=106 \mathrm{Mpc}\right.$ for $\left.\mathrm{H}_{0}=70 \mathrm{~km} \mathrm{~s}^{-1} \mathrm{Mpc}^{-1}\right)$. It is the host galaxy of one of the largest known radio sources in the sky: the radio lobes extend for $1^{\circ} .2$ (over $2 \mathrm{Mpc}$, Waggett et al. 1977, Readhead et al. 1978, Cohen and Readhead 1979, Jones et al. 1986, Jones and Wehrle 1994).

HST WFPC2/PC images of NGC 6251 , obtained in the broad band filters F547M $(\sim V)$ and F814W $(\sim I)$, show a dust disk, $1^{\prime \prime} .43(680 \mathrm{pc})$ across and inclined by $\sim 14^{\circ}$ with respect to the line of sight (Figure 1 , Ferrarese et al. 1998). The minor axis of the disk is misaligned with respect to both the minor axis of the galaxy, and the axis of the parsec scale radio jet. Assuming a Galactic value of the surface density of hydrogen to the color excess (Bohlin et al. 1978) the disk mass is $\sim 3 \times 10^{6} \mathrm{M}_{\odot}$.

Additional narrow band images bracketing the $\mathrm{H} \alpha+[\mathrm{NII}]$ emission, show that a resolved region of ionized gas is present within the disk. HST/FOS spectra were obtained with $4.85 \AA$ resolution in the $6270-8500 \AA$ wavelength region at the seven positions shown in Figure 1, using the $0^{\prime \prime} .09$ square aperture. The velocities of the ionized gas at the seven locations are compared with a model in which the gas is confined in a disk in Keplerian rotation around a central mass (see Ferrarese et al. 1998 for additional details). Free parameters in the model are the inclination, location (with respect to the aperture grid) and position angle of the Keplerian disk, as well as the mass $M_{B H}$ of the central point source. The best fit to the data points, shown in Figure 2, is obtained for $M_{B H}=(8.7 \pm 2.5) \times 10^{8} \mathrm{M}_{\odot}$. Fits to the data which do not include a central point source or assume that the 

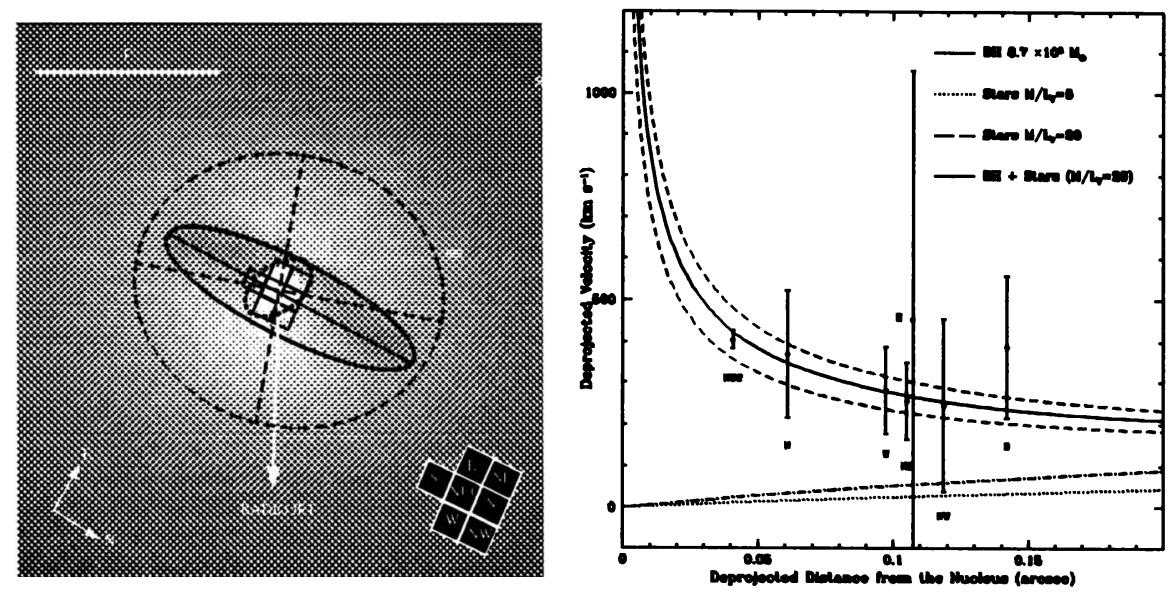

Figure 1 (left). $2^{\prime \prime} .7 \times 2^{\prime \prime} .7$ section of the F547M image of NGC 6251 . The larger ellipse is the best fitting isophote at that radius, while the smaller solid contour represents the outer edge of the dust disk. The best kinematical model, with parameters given in Figure 2, predicts that the ionized gas is confined in the disk shown by the inner, dotted line. The inclination of the disk is consistent with the inclination of the parsec scale jet (Jones and Wehrle 1994, Birkinshaw and Worrall 1993), and the major axis of the disk is misaligned with respect to the outer dust disk, indicating that the gas-dust structure is warped.

Figure 2 (right). The dotted, and dash-dotted lines show the expected rotation curve due to the stellar component for $(M / L)_{V}=5$ and $(M / L)_{V}=20$ respectively. The thin solid line is the Keplerian rotation due to a $8.7 \times 10^{8} \mathrm{M}_{\odot}$ black hole. The thick solid rotation curve is obtained when the contribution from the stars (for $(M / L)_{V}=20$ ) is added. The dashed lines represent the $1 \sigma$ uncertainty on the final velocity due to the uncertainty in the black hole mass $\left( \pm 2.5 \mathrm{M}_{\odot}\right)$.

gas is in a bi-directional outflow are excluded at over the $99 \%$ confidence level. Given the value of $M_{B H}$, we find $(M / L)_{V} \sim 650 \mathrm{M}_{\odot} / \mathrm{L}_{\odot}$ inside 0 " $n 9$ $(=46 \mathrm{pc})$. This, together with the presence of radio activity, suggests that the majority of the central mass is concentrated in a nuclear black hole.

LF acknowledges support from NASA grant HF-01081.01-96A

\section{References}

Birkinshaw, M. \& Worrall, D.M. 1993, Ap.J. 412, 568

Bohlin, R. et al. 1978, Ap.J. 224, 132

Cohen, M.H. \& Readhead, A.C.S. 1979, ApJ, 233, L101

Ferrarese, L. et al. 1998, in preparation

Jones, D.L. et al. 1986, ApJ, 305, 684

Jones, D.L. \& Wehrle, A.E. 1994, 427, 221

Readhead, A.C.S. et al. 1978, Nature, 272, 131

Waggett, P.C. et al. 1977, MNRAS, 181, 465 